Temperature controlled interval contact design for ultrasound assisted liquid-liquid extraction

Peer-reviewed author version

John, Jinu Joseph; Kuhn, Simon; BRAEKEN, Leen \& Van Gerven, Tom (2017)

Temperature controlled interval contact design for ultrasound assisted liquid-liquid extraction. In: CHEMICAL ENGINEERING RESEARCH \& DESIGN, 125, p. 146-155.

DOI: 10.1016/j.cherd.2017.06.025

Handle: http://hdl.handle.net/1942/26452 
See discussions, stats, and author profiles for this publication at: https://www.researchgate.net/publication/317948608

Temperature controlled interval contact design for ultrasound assisted liquidliquid extraction

Article in Chemical Engineering Research and Design · June 2017

DOI: 10.1016/j.cherd.2017.06.025

CITATIONS

4 authors, including:

ค. Jinu Joseph John

University of Leuven

5 PUBLICATIONS 20 CITATIONS

SEE PROFILE

Tom Van Gerven

University of Leuven

218 PUBLICATIONS $\quad \mathbf{4 , 2 9 4}$ CITATIONS

SEE PROFILE
Simon Kuhn

University of Leuven

58 PUBLICATIONS 769 CITATIONS

SEE PROFILE

Some of the authors of this publication are also working on these related projects:

KULeuven PhD grant View project

Project $\quad$ Process Intensification for Rare Earth Element Recovery from bauxite residue View project 


\title{
Temperature controlled interval contact design for ultrasound assisted liquid-liquid extraction
}

\author{
Jinu Joseph John ${ }^{1}$, Simon Kuhn ${ }^{1}$, Leen Braeken ${ }^{2}$, Tom Van Gerven ${ }^{1}$ \\ ${ }^{1}$ Process Engineering for Sustainable Systems (ProcESS), Department of Chemical Engineering, KU \\ Leuven, Leuven, Belgium \\ ${ }^{2} \mathrm{KU}$ Leuven Lab4U - Faculty of Industrial Engineering, Agoralaan Building B, B-3590 Diepenbeek, \\ Belgium
}

\begin{abstract}
This work aims at constructing a design which integrates direct contact method (or a solid contact interface) with temperature control for chemical process applications. To realise this integration a two-step approach is proposed. Firstly temperature control is introduced by is achieved by suspending the tubing in a temperature controlled and sonicated liquid medium (indirect contact) and secondly the direct contact elements are introduced at regular intervals along the tubing. This design which incorporates both direct and indirect approaches of ultrasound transfer is termed the hybrid contact reactor. Two feasible configurations, open and closed intervals, were assessed. The hybrid reactors performed better than the indirect contact reactor (20 to $27 \%$ higher yield) for lower residence times of $<45 \mathrm{~s}$ and similar for higher residence times. Comparing the two hybrid designs, even though their performance was similar the closed interval gave more stable and distinct yields. The latter design was scaled up to 10 times in volume with a $2 \mathrm{~mm}$ ID tube design. This design showed a relative performance similar to the interval contact design which gave the highest yields thus far for the same operating conditions.
\end{abstract}




\section{Introduction}

In the last decade, ultrasound has gained momentum in relation with micro- or milli-structured devices for the use in chemical processes (Fernandez Rivas et al., 2012; Fernandez Rivas and Kuhn, 2016). This interest originates from its ability of being a non-contact source to activate or initiate physical (Cravotto and Cintas, 2012, 2009, 2006; Katou et al., 2005; Linares et al., 1987; Okadap et al., 1972; Suslick et al., 1990) or chemical effects (Cravotto and Cintas, 2006; Leonelli and Mason, 2010; Mason, 2000; Suslick et al., 1990). These effects are a result of the cavitation induced by the ultrasound in the liquid medium and are documented to contribute to many factors such as improved mass transfer characteristics (Ahmedomer et al., 2008; Monnier et al., 2000, 1999; Okadap et al., 1972). This mass transfer improvement is of particular interest, as the chemical process of focus is liquid-liquid extraction. The efficiency of such a process mainly depends on the effectiveness of contact between the two immiscible phases. In batch systems, this contact depends on the agitator performance, but in a microchannel, this in turn depends on the flow pattern generated and the innovative physical structures implemented (Dessimoz et al., 2008; Kashid et al., 2011, 2005; Kashid and Agar, 2007; Plouffe et al., 2014; Xu et al., 2013). The generated flow pattern is a result of the fluid properties and the process and design parameters implemented (Dessimoz et al., 2008; Di Miceli Raimondi et al., 2014; Kashid and Kiwi-Minsker, 2011; Kashid and Agar, 2007). Improving mass transfer characteristics at a fixed operating condition might prove challenging without active intervention. To this effect, ultrasound can prove useful as it can provide active mixing effects by cavitation; for example, by the vibration or collapse of a cavitation bubble under the influence of the acoustic pressure field. In addition, when immiscible liquid-liquid systems are sonicated it has been observed that the cavitation disturbs the interface causing jetting of one phase into the other resulting in the formation of emulsions or micro droplets of one phase in another. This can provide additional interfacial area required for mass transfer and hence improve the extraction process.

One of the key factors that contribute to an efficient ultrasound assisted processes is the effective transmittance of ultrasound to the required region of operation or system. The transmittance determines the amount of the power that needs to be supplied and this in turn affects economic aspects of the process. Hence, the biggest challenge when using ultrasound is to effectively combine ultrasound with a microreactor setup. Currently, the most common methodology is to have the microreactor setup suspended in an ultrasonic bath (Ahmedomer et al., 2008; Aljbour et al., 2009). There are many disadvantages for this method namely poor transmission of power and equipment specific performance dependency. A versatile approach for a more efficient transfer of the ultrasound power is to create a direct contact between the 
ultrasound-irradiating device and the reaction mass, thus minimizing or even eliminating the transfer media in terms of number and thickness. Therefore, a direct contact reactor was previously designed (John et al., 2016a) by placing the tubing of $0.8 \mathrm{~mm}$ I.D (internal diameter) and $1.6 \mathrm{~mm}$ O.D (outer diameter) into a channel cut through a metal plate and having the plate bolted onto the ultrasound transducer. This design proved fruitful for the operating and process conditions explored with the best results obtained at low frequency of $20.3 \mathrm{kHz}$ and high amplitude of $840 \mathrm{mV}$. These conditions resulted in an yield improvement 50 to $60 \%$ for a residence time of less than 87 seconds. Subsequently, an improvement to the direct contact design was proposed and then verified by the addition of intervals along the channel; named as the interval contact reactor(John et al., 2016b). Intervals are nothing but contact elements of $1 \mathrm{~mm}$ thickness provided along the length of the channel through which the ultrasound is transferred to the tubing. These elements help by better focusing the intensities along the channel to the tube, When both designs were compared at similar operating conditions of 20.3 $\mathrm{kHz}$, amplitude of $590 \mathrm{mV}$ and input power of $20 \mathrm{~W}$, the interval design showed an improvement of 13 to $23 \%$ over the direct contact design.

The major limitation with the direct contact and the interval design is the inability of an accurate temperature control during sonication as it is a major requirement in most chemical reactions. Also when studying the direct and interval designs the temperature differences between the inlet and outlet of the interval reactor design range between 4 to $12{ }^{\circ} \mathrm{C}$. Even though it was proved that these temperature differences are insufficient to bring about improvement in yield as brought about by ultrasound, there might be a minor contribution by it. To understand the contribution of ultrasound alone temperature control needs to be implemented. In spite of all the limitations that the classical ultrasonic bath possesses, due to the presence of the liquid medium there is a possibility for temperature control by using a thermostatic control element. This research aims at incorporating the temperature control approach of an ultrasonic bath with the direct contact methodology, to arrive at a hybrid design for use in chemical processes.

In addition to temperature control, for implementation in chemical industry scalability is an important factor. Due to the small penetration depth of the ultrasound, the processes that are studied in combination with it are often limited to either micro- or milli-scale (Ahmed et al., 2006; Aljbour et al., 2009; Hübner et al., 2012; Monnier et al., 2000). However, in the different research reports published earlier the scalability is explored with a variety of different designs and configurations (Cravotto and Cintas, 2006; Gogate and Pandit, 2004; Leonelli and Mason, 2010). In the case of the microreactor, one approach to scale up is numbering up (Kashid et al., 2010). To implement this, it is important that the potential of the system numbered up be exploited to the maximum. In this research, we attempt to scale up the hybrid design in terms of the diameter that is feasible in the design for best possible throughput. 


\section{Materials and methods}

\subsection{Reactor Design}

Two intrinsic elements need to be incorporated into the original design of the direct contact reactor, they are temperature control and direct contact elements. In this research, the temperature control is first established by constructing a mini-ultrasonic-bath configuration matching the geometrical parameters of the direct contact reactor (John et al., 2016a) and then direct contact elements are installed into it as intervals. Therefore, the implementation is via two consecutive steps with reactor plates made in succession.

The introduction of temperature control by a matching mini-ultrasonic-bath consists of a 10 $\mathrm{mm}$ thick, $80 \times 80 \mathrm{~mm}$ aluminium plate, hollowed out in the centre to a depth of $8.5 \mathrm{~mm}$ to supply the temperature-control medium. This medium is pumped through two elbows with push-in fittings provided at both ends of the hollowed-out area, which act as inlet and exit points. The reaction mass is pumped through a 1/16" O.D. (0.8 mm I.D) PFA (Perfluoroalkoxy) tubing, suspended between the ends of the hollow as shown in Figure 1. The eight tube ends are each kept watertight with a 1/4" fitting. The tubing position matches the design characteristics of the direct contact reactor regarding distance from the transducer and sonicated length (John et al., 2016a, 2016b). A $5 \mathrm{~mm}$ plexiglas plate is screwed onto the aluminium plate forming a watertight compartment to conduct the temperature control medium. The entire assembly is bolted onto a transducer as shown in Figure 1.

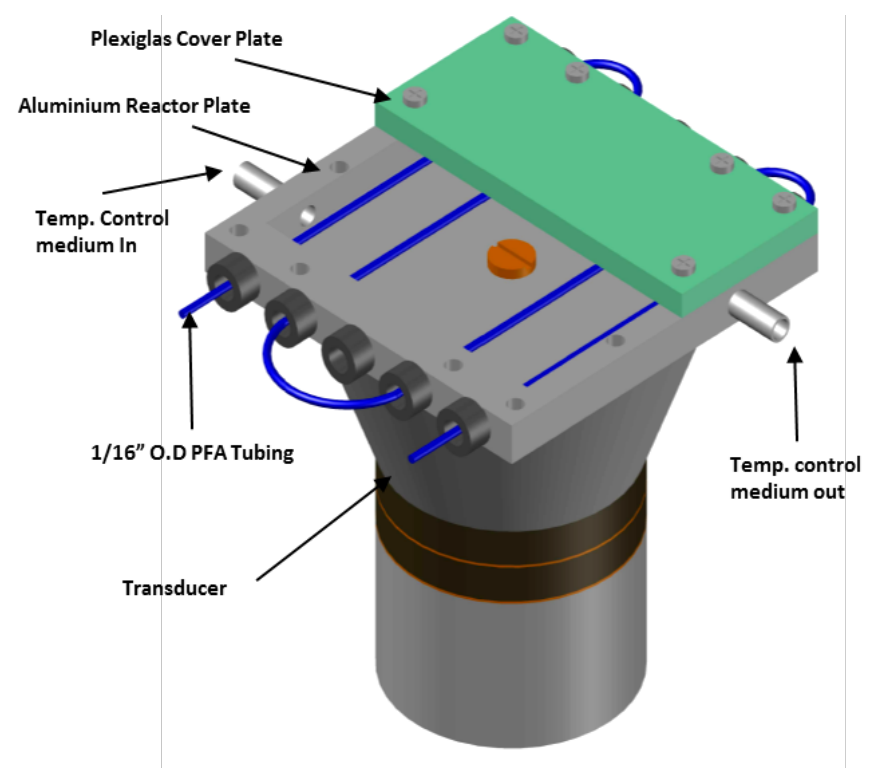

Figure 1: Indirect contact type reactor assembly

The temperature-control medium also facilitates transfer of the ultrasound from the transducer to the reaction mass. In the direct contact and the interval contact the ultrasound is transmitted 
directly from the plate to the tube but in this design it is through a liquid medium thus providing an indirect contact between the source and the sonicated system. This reactor is therefore named the indirect contact reactor. This method of sonication holds an advantage over conventional horn setups as it prevents the reaction mass coming in direct contact with the sonication source, thereby avoiding metal contamination by erosion through ultrasound source surface cavitation.

The mini-bath approach does not yet incorporate the direct contact methodology. The direct contact is introduced by providing intermittent contact elements along the tubing length. This is achieved by providing metallic elements of $1 \mathrm{~mm}$ thickness, perpendicular to the tube within the hollowed region where the tubing is suspended (see figure 2 (c)). This design is termed as the hybrid contact design as it utilizes the direct contact of the interval contact design and the indirect methodology of transfer by a liquid medium in an ultrasonic bath. The two different designs of incorporating the interval contact are named the hybrid open-interval reactor and the hybrid closed-interval reactor. They are as shown in Figure 2 (a) \& (b), respectively. The above mentioned configurations s were studied, as the open interval is a representation of the interval contact design and it was unsure whether a proper contact be maintained as unlike it the tubing is not held in place by a cover plate but by fittings at the ends of each pass of tubing. In this regard the closed interval design was constructed to compare the effectiveness or the need for a more robust contact. . 


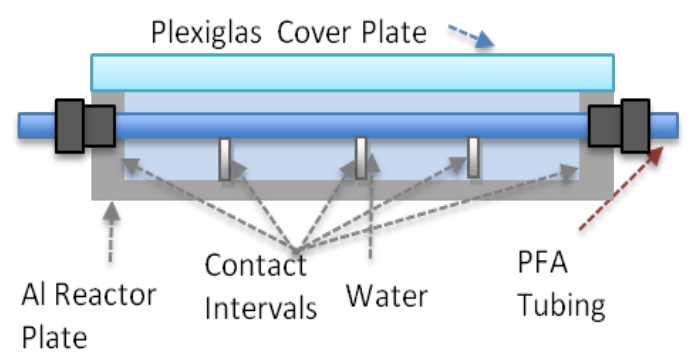

(a)

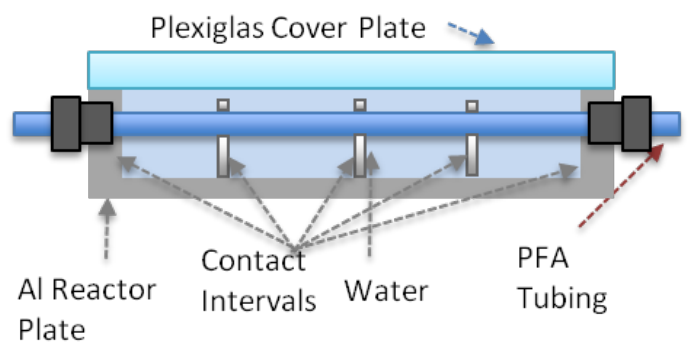

(b)

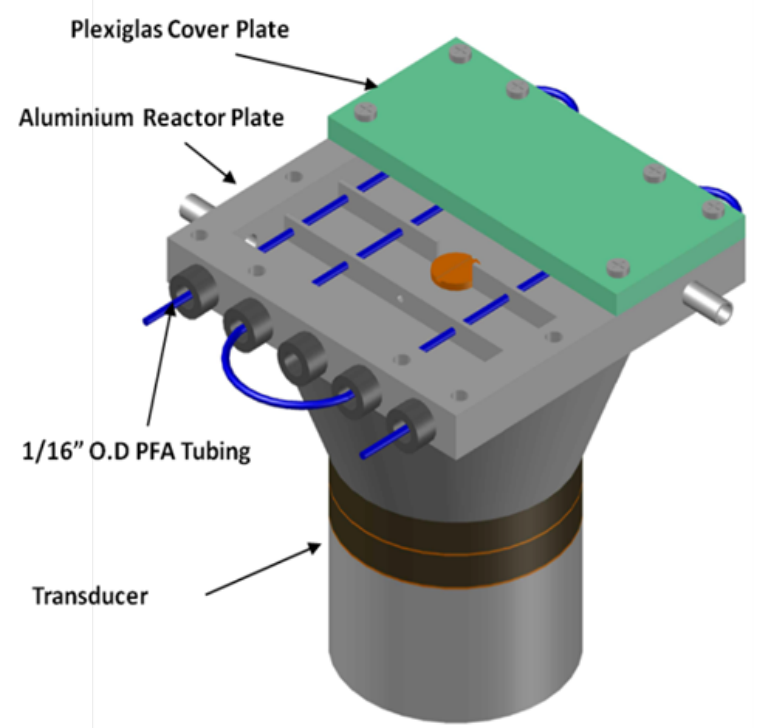

(c)Figure 2: The hybrid closed-interval reactor: (a) cross section of open-interval reactor, (b) cross section of closed-interval reactor, (c) assembled reactor for $1 / 16$ " OD tube

The number of intervals was varied and studied in a previous publication (John et al., 2016b) and five intervals per channel was found to be the best in terms of percentage yield obtained from the two-phase reaction. The ends suspending the tube are to act as two intervals and the remaining three intervals are arranged as either with tube passing over or through the interval for the open- and closed-interval reactor, respectively.

\subsection{Experimental setup}

The designed reactor assembly is connected to two ProSense NE-300 syringe pumps, to pump the organic and the aqueous phase. The pumps are connected to the reactor via a PTFE T-junction to generate the required flow pattern, which in this case is a slug flow. The 
temperature of the temperature-control medium (water) is maintained with a Lauda ECO RE 415 thermostatic bath. The outlet of the tubing coming from the reactor plate is connected to a Zaiput SEP 10 membrane separator. The membrane used is PTFE membrane of $0.5 \mu \mathrm{m}$ pore size. A Photron UX 100 high speed camera is used to study the behaviour of the twophase flow in the tubing with and without sonication.

The transducer connected to the reactor is a multi-frequency type, which can operate at frequencies of 20, 40 and $60 \mathrm{kHz}$ (Ultrasonics World MPI-7850D-20-40-60 H). The transducer is connected to a Picotest G5100A waveform generator through an E\&I 1020L RF amplifier. The waveform generator controls the characteristics of the ultrasound wave like frequency, amplitude and shape of the wave. The signal generated is then amplified from $\mathrm{mV}$ to volts by the amplifier. The transducer is kept from overheating due to prolonged use by the use of a ventilation fan. To obtain resonance frequencies the setup is connected with an impedance analyzer (Sinephase 16777k) instead of the waveform generator and amplifier.

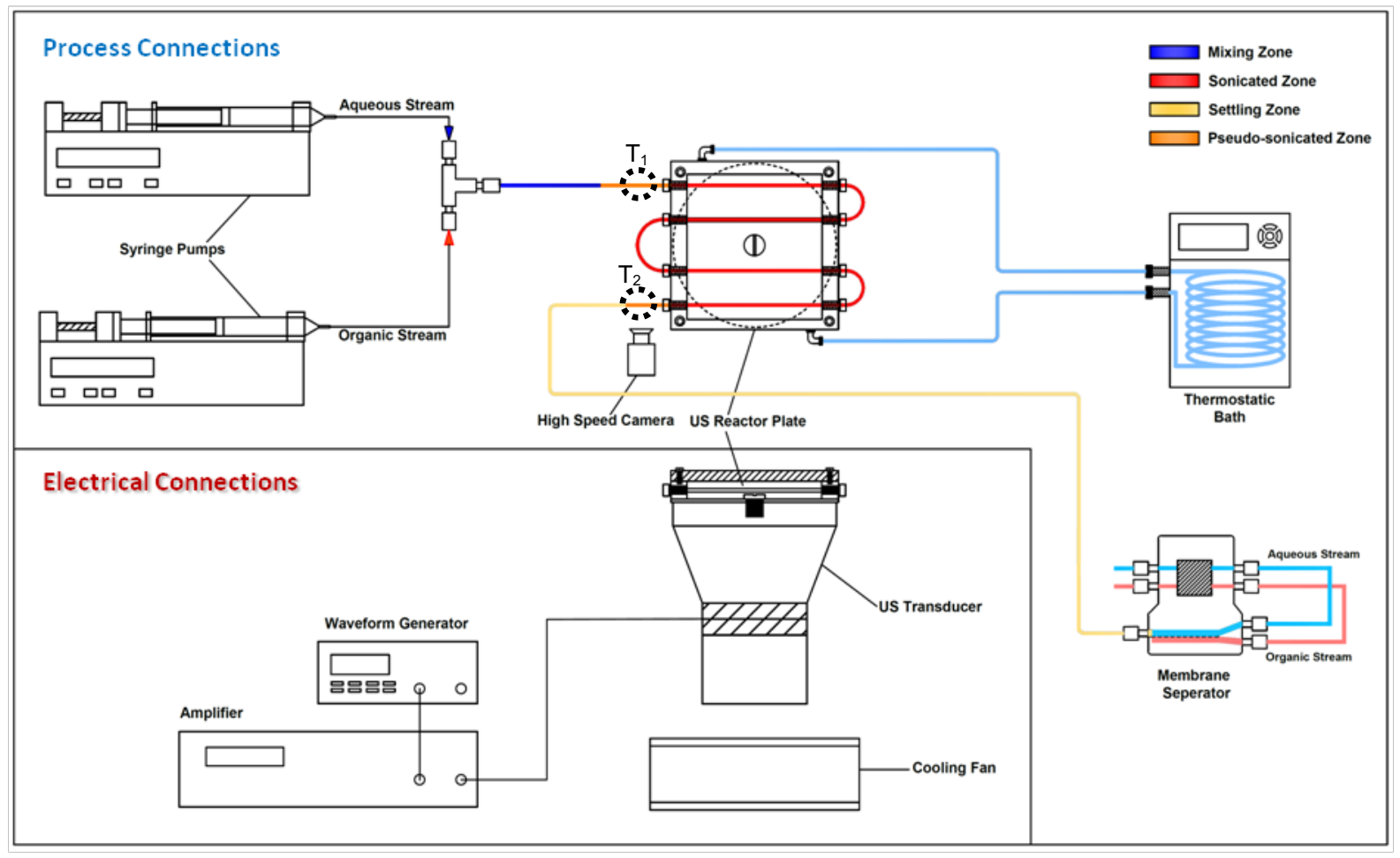

Figure 3: Experimental setup

To understand the power transferred by the ultrasound temperature measurements are performed at the inlet and outlet of the reactor plates denoted by position $T_{1} \& T_{2}$ in Figure 3 . The temperature measurements are performed by incorporating a T-junction at these points with a thermocouple inserted in one of the ends. The thermocouple is connected to a PICO temperature logger connected to a computer. 


\subsection{Reaction}

To understand the effect of ultrasound on an extraction process, an appropriate extraction process needs to be selected. The same two-phase system used in the direct contact reactor plate type was used, which is the hydrolysis of p-nitrophenyl acetate (John et al., 2016a, $2016 \mathrm{~b})$. The compound dissolved in toluene at a concentration of $0.05 \mathrm{M}$ acts as the organic phase and $0.5 \mathrm{M}$ sodium hydroxide solution is used as the aqueous phase.

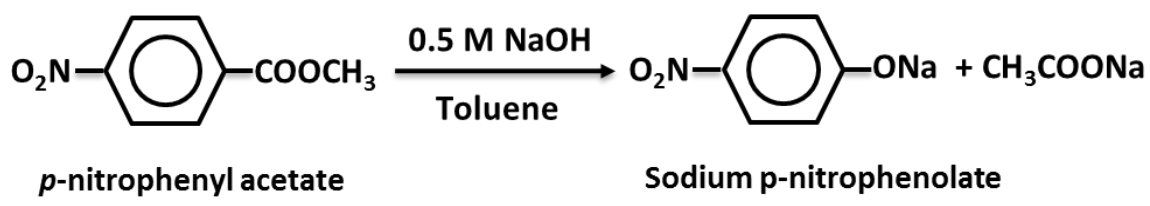

Figure 4: Hydrolysis of $p$-nitrophenyl acetate

The concentration of the product formed is determined by the formation of the yellow colour, which is a result of the sodium $p$-nitrophenolate being dissolved in water. The intensity of the colour $\left(\lambda_{\max }=400 \mathrm{~nm}\right)$ is analysed by a spectrophotometer (Shimadzu UV-1601). All experiments are performed in triplicate and the average is taken. The error ranges for each point are represented as the standard deviation of these experiments.

\section{Results and Discussion}

\subsection{Effect of Indirect Contact}

The behaviour of the two-phase flow in the indirect contact reactor is first evaluated. The qualitative examination of the initiation mechanism with a high-speed camera revealed cluster initiation as the initiation mechanism of emulsion formation (John et al., 2016a). This is the same initiation mechanism as the direct contact design implying the change of the method of application of ultrasound does not affect the initiation mechanism. One of the aims of this design is temperature control; this is assessed by measuring the temperature differences between inlet and outlet of the tube entering and exiting the reactor plate. This is done by attaching thermocouples through a T-junction at these points. The measurements are done with water as the flow through medium. These experiments were performed at the same flow rates $(0.1,0.2,0.3,0.5,0.7 \& 0.9 \mathrm{ml} / \mathrm{min} \approx$ residence time ranges $<87 \mathrm{~s})$, frequency $(20.3$ $\mathrm{kHz})$ and power $(20 \mathrm{~W})$ conditions as the direct $\&$ interval contact design. This temperature differences obtained where in the range of 0.9 to $0.2{ }^{\circ} \mathrm{C}$. The higher temperature differences (4 to $12{ }^{\circ} \mathrm{C}$ ) were observed for the direct and interval design as the fluids were only heated by the ultrasound. In this design the difference is low as there is simultaneous heating by the 
ultrasound and cooling by the temperature control medium. As the differences obtained are minimal, this design may prove useful to assess accurately the independent influence by ultrasound,

Having established temperature control the design is examined quantitatively in terms of the yield of the two-phase reaction. The effect of the variation of frequency is first studied at a constant total flow rate of $0.2 \mathrm{ml} / \mathrm{min}$, amplitude of $590 \mathrm{mV}$ and a net applied power of $20 \mathrm{~W}$ (which is the difference between the forward and reflected power). For these experiments, the forward power and reflected powers were kept constant at $41 \pm 1$ and $21 \pm 1 \mathrm{~W}$ (measured by the amplifier), respectively. The temperature-control medium is maintained at a temperature of $20^{\circ} \mathrm{C}$. The results obtained are plotted in Figure 5 .

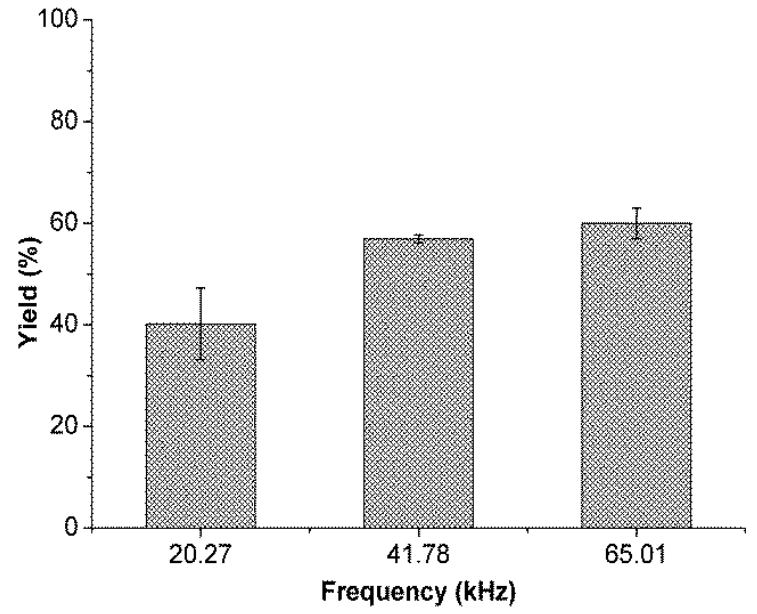

Figure 5: Variation of yield with frequency at constant amplitude $(590 \mathrm{mV})$, flow rate $(0.2 \mathrm{ml} / \mathrm{min})$ and net applied power $(20 \mathrm{~W})$ : Indirect contact reactor

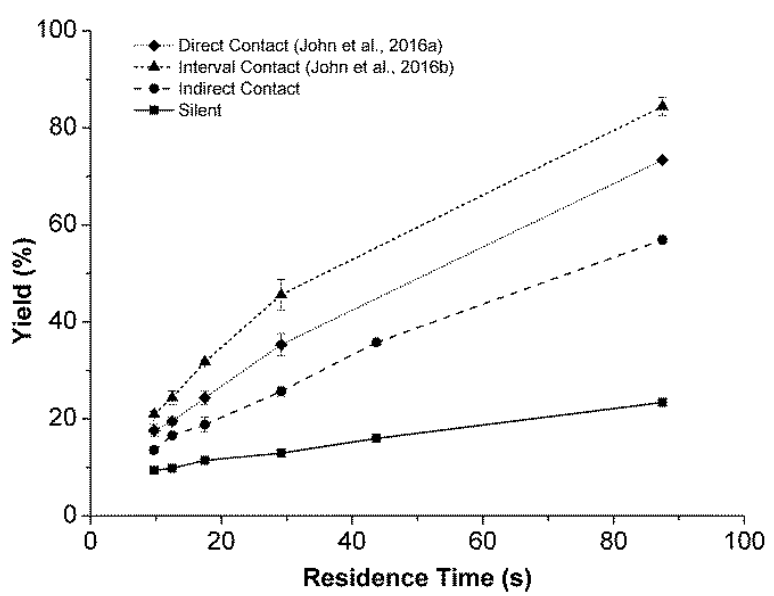

Figure 6: Comparison of the yields between the indirect contact reactor and the direct contact types.

It was shown earlier for the direct contact design (John et al., 2016a) that the yields decreased with increasing frequency. However, for the indirect contact design an inverse behaviour is observed with the 41.78 and $65.01 \mathrm{kHz}$ frequency showing similar yields, as shown in Figure 5. To understand this behaviour, the temperature difference between the inlet and outlet of the sonicated tubes was measured with water for the same operating conditions as an indication of the calorimetric powers transferred at the different frequencies for this reactor arrangement. The differences in temperature obtained were $-0.090,-0.021$ and $-0.026{ }^{\circ} \mathrm{C}$ for the $20.27,41.78$ and $65.01 \mathrm{kHz}$ frequencies, respectively. The difference was also negative as the liquid entering the reactor was in the range of $23-24^{\circ} \mathrm{C}$, while the reactor is maintained at $20^{\circ} \mathrm{C}$. The temperature differences obtained are very small in comparison to the direct and interval contact designs, as a temperature-controlled fluid is used here as the transfer medium and due to the large heat transfer area of the tubing which helps maintains the temperature 
effectively. The results show that the reaction fluids are heated almost equally at 41.78 and $65.01 \mathrm{kHz}$, and more than at $20.27 \mathrm{kHz}$. These temperatures differences, even though small, reflect the intensities of the power transferred at these frequencies, which shows that higher intensities are transferred at the higher frequencies in comparison to the lower frequency studied. These findings indicate similar behaviours with the yields, indicating a shift in frequency of choice from $20 \mathrm{kHz}$ range to the $40-65 \mathrm{kHz}$ range when the transfer occurs through a liquid medium. This shift is attributed to the change in resonance frequencies because of the presence of an entirely new composition of materials to which the sonication is applied.

To have an understanding of the effect of the indirect contact design in comparison to the direct contact designs, the yields obtained for similar flow rates were examined. The direct contact designs were compared to the $41.78 \mathrm{kHz}$ as the error ranges were smaller at that frequency and because it was difficult to maintain the emulsification for all the flow rates examined at the $65.01 \mathrm{kHz}$ frequency. The amplitude and net applied power were kept constant during the comparison at $590 \mathrm{mV}$ and $20 \mathrm{~W}$, respectively. The results obtained are as shown in Figure 6, showing the interval contact to be still the best for all the flow rates examined. The indirect contact reactor is at the lowest in terms of yield, but still higher than the silent condition, thereby indicating a room for improvement, which may be alleviated by the presence of the direct contact elements.

\subsection{Effect of introduction of direct contact element}

Having established a baseline in behaviour by the indirect approach, the effect that the interval has on the sonication of the two-phase flow is further studied qualitatively using two different designs open and closed interval. The first observations were made with reference to the initiation of the emulsion. The initiation observed at the entry of the tubing into the sonicated area or the reactor plate is shown in Figure 7. 


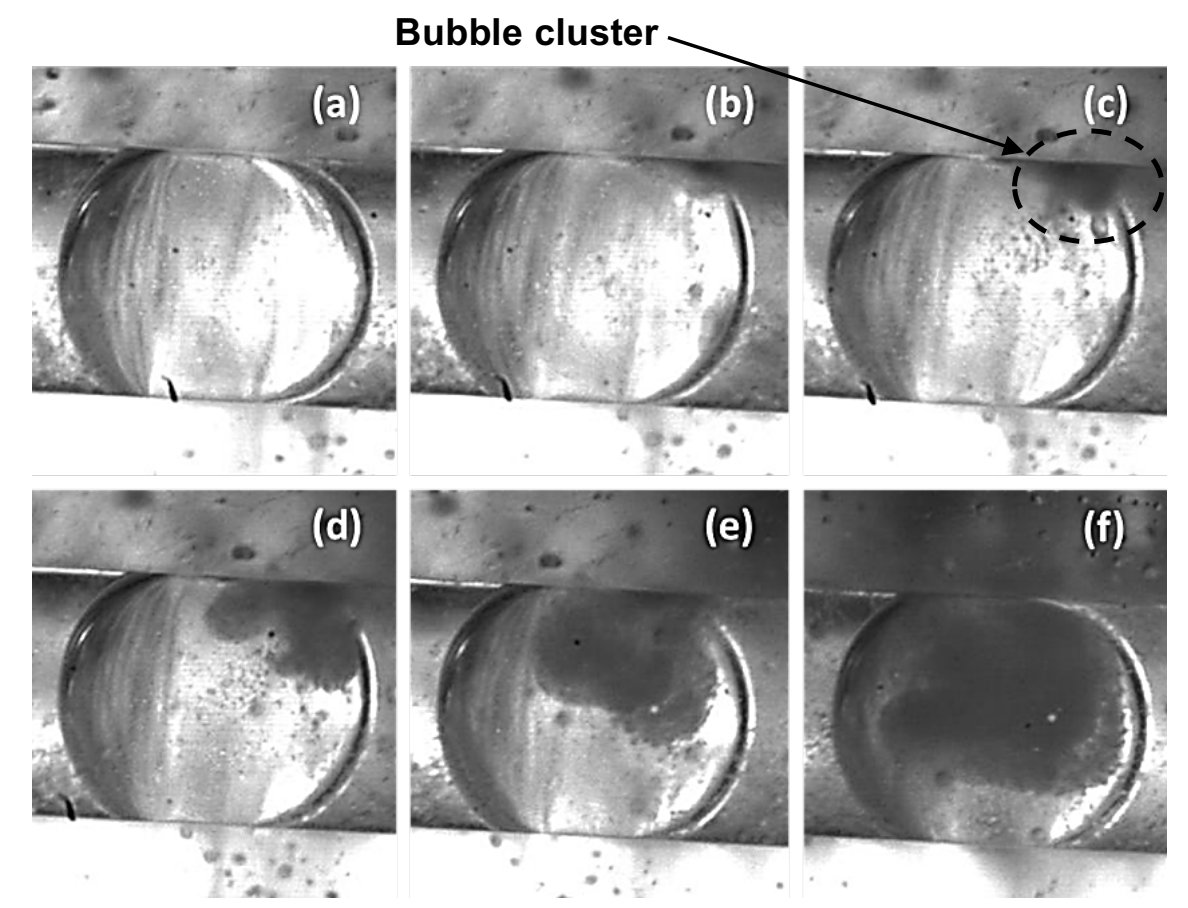

Figure 7: Formation of the emulsion in the aqueous slug in $0.8 \mathrm{~mm}$ hybrid reactor(a to $f \approx 0.033 \mathrm{~s}$ ). This image is taken at the entry between first and second interval.

Figure 7 shows the formation of a cluster of bubbles at the top interface and, hence, indicates that the mechanism is by cluster initiation and shows a lack of large bubble formation. The emulsion propagates from the interface and progresses inwards into the dispersed bubble which is the aqueous phase in this case. This is similar to what was observed for the indirect contact design and hence, the intervals do not really affect the initiation mechanism. Having established the initiation mechanism, the two phase system was studied at the intervals and mid-intervals to see the behaviour exhibited by the emulsified slugs or dispersed bubble. 


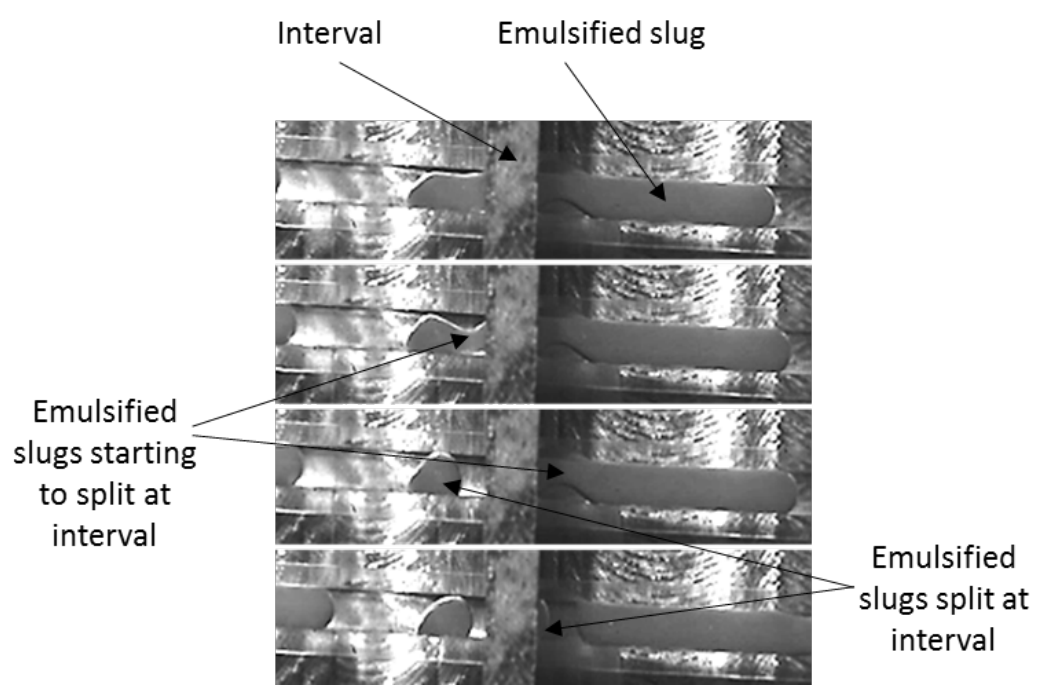

(a)

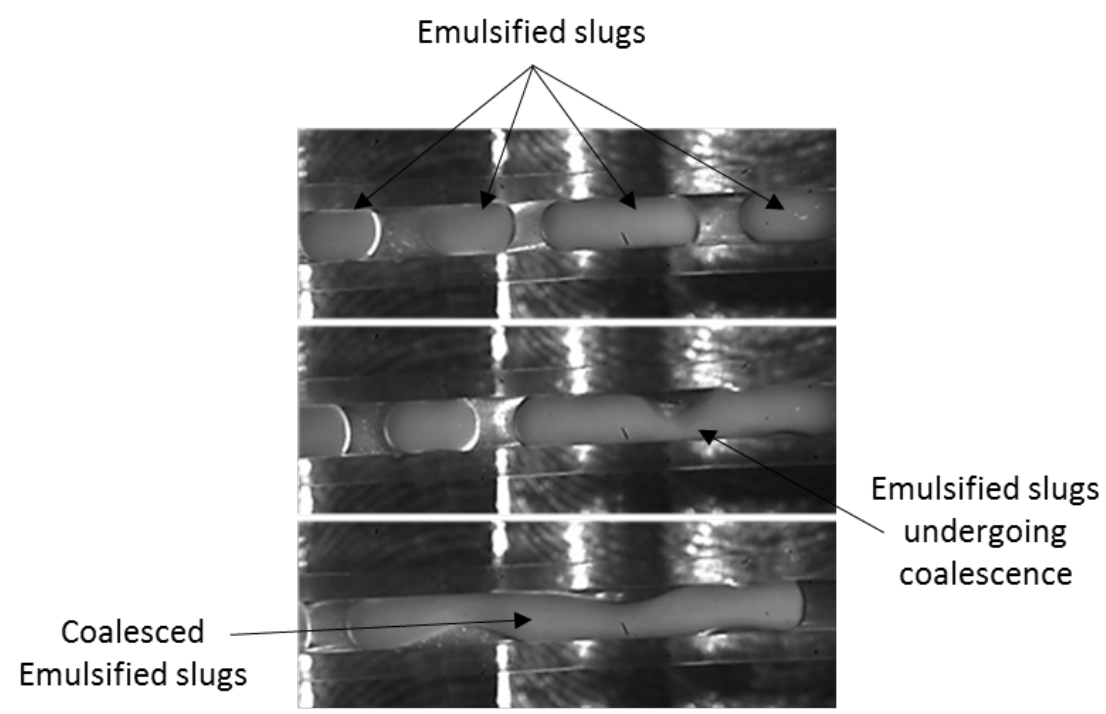

(b)

Figure 8: (a) Splitting of the slugs at the interval (b) consolidation of bubble between the interval

As observed in figure 8 there is a dynamic change in the emulsified slug's sizes at the intervals and between the intervals. The majority of the reduction of the emulsified slug sizes seems to be concentrated at the location of the intervals and a majority of the coalescence of the emulsified slugs seems to be concentrated in between the intervals. This behaviour is observed for both designs.

To achieve a quantitative analysis of the effect of the different hybrid designs, the first set of experiments was conducted by varying the frequency. All experiments were conducted with the temperature control medium maintained at $20^{\circ} \mathrm{C}$. Even though the transducer used is a multi-frequency transducer for the frequencies 20,40 and $60 \mathrm{kHz}$ ranges, the hybrid designs coupled with this transducer show multiple resonance frequencies ranges when examined with an impedance analyser. The frequencies were examined at constant conditions of 
amplitude $(590 \mathrm{mV})$ and net applied power $(20 \mathrm{~W}=$ forward power of $41 \pm 1 \mathrm{~W}$ - reflected power of $21 \pm 1 \mathrm{~W}$ ) for the same flow rate of $0.1 \mathrm{ml} / \mathrm{min}$. The frequencies that meet the specified power conditions and the corresponding yields obtained are shown in Figure 9.

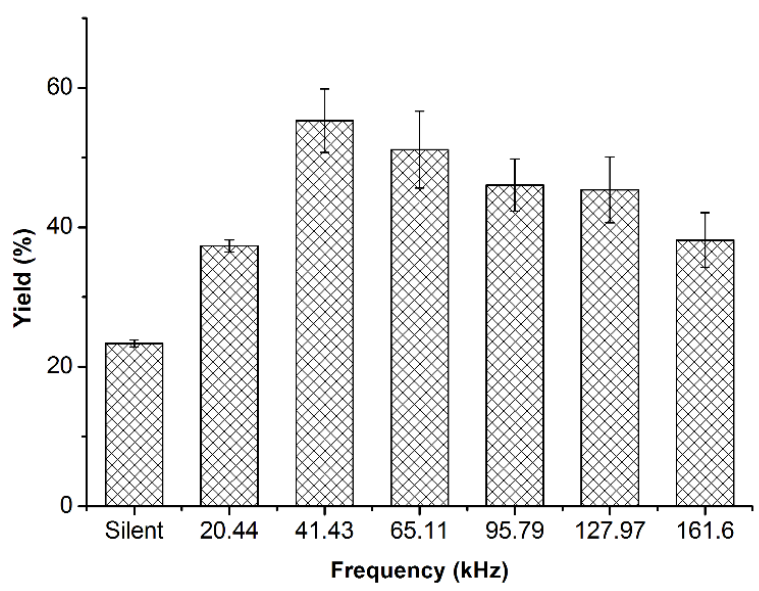

(a)

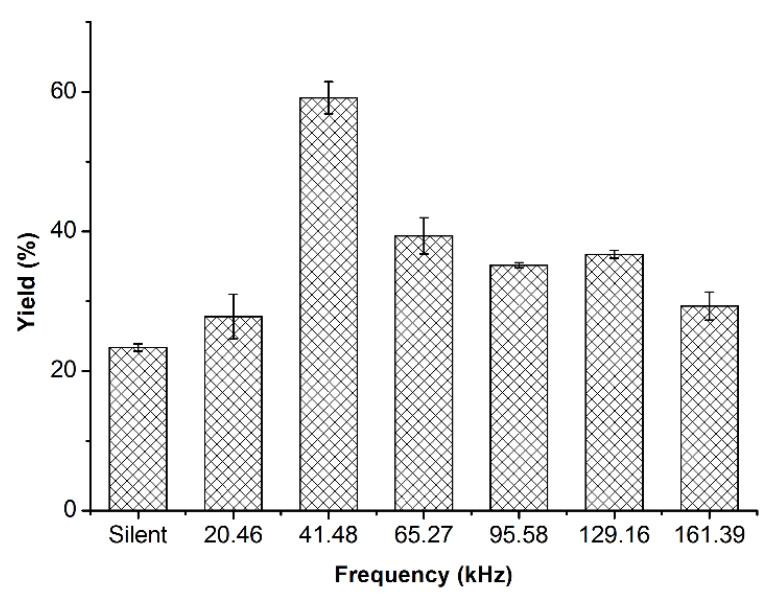

(b)

Figure 9: Variation of yields with frequency at contact amplitude of $590 \mathrm{~m} . \mathrm{V}$, flow rate of $0.1 \mathrm{ml} / \mathrm{min}$ and net applied power of 20 $W$ for (a) Open interval design (b) closed interval design

From both the figure $9(\mathrm{a})$ and $9(\mathrm{~b})$ it is seen that multiple resonance frequencies ranging up to $161 \mathrm{kHz}$ are obtained for designs. It is an improvement in terms of the range of frequencies studied from the indirect contact design, which was limited to $65 \mathrm{kHz}$. The frequency points at same power input are similar but not exactly the same, this is attributed the differences in the sonicated structure brought about by the minor changes (open \& closed interval) in the design. Comparing both the designs, the open interval seems to have better results over a wider range of frequencies than the closed interval. But when examining the point of the highest yield which is the $40 \mathrm{KHz}$ range the highest average value is obtained for the closed interval. Also comparing the error ranges for both the designs, the open interval has the largest. This large error ranges can be a result of the open interval design, wherein the tubing is passing over the interval. The closed interval design is advantageous in this sense because applying ultrasound can impart a pressure at the point of contact between the interval and tubing, causing the tubing to be misplaced. When the tubing is just resting on top of the interval, as is the case of the open interval design, it can result in an increased probability for loss of contact on sonication, thereby causing increased fluctuations in the results and this is observed in the experiments.

Having determined the best operating frequency, the hybrid designs are studied for the variation in flow rates in comparison to the indirect contact reactor. Similar operating conditions are imposed on the different designs wherein the individual flow rates are varied from 0.1 $\mathrm{ml} / \mathrm{min}$ to $0.9 \mathrm{ml} / \mathrm{min}$ at a ratio of $1: 1$, the amplitude is kept at $590 \mathrm{mV}$ and the net applied 
power at $20 \mathrm{~W}$. The results obtained are plotted in Figure 10 in terms of the corresponding residence times.

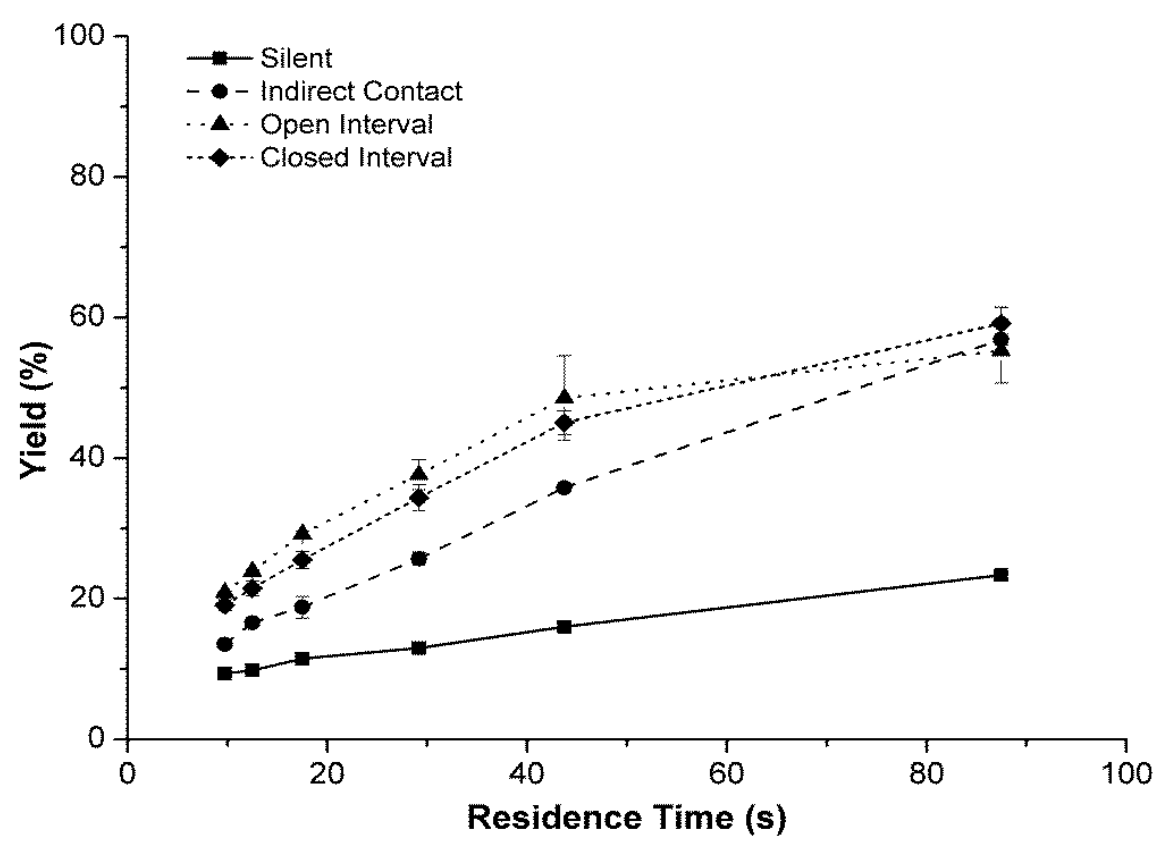

Figure 10: Variation of yield (\%) with residence times for change of Interval type change at a frequency of $41.4 \mathrm{kHz}$, amplitude of $590 \mathrm{mV}$ and net applied power of $20 \mathrm{~W}$.

Figure 10 shows an increase in yield upon sonication for all the designs when compared to the silent condition. Comparison of the sonicated results at the highest residence time shows similar results, indicating there is little or no improvement by the presence of the direct contact elements. Also it is seen that above the $45 \mathrm{~s}$ residence time the linearity in the graph is disturbed and this is hypothesised to be a result of the coalescence behaviour brought about by the intervals. At the higher residence time, the coalescence probably decreases the net interfacial area available for mass transfer, thereby decreasing the yield in the interval designs. The other residence times show improved and comparable yields for the hybrid designs in comparison to the indirect contact design. The improvement in the yield by the hybrid design is in the range of 20 to $29 \%$ in comparison to the indirect contact reactor, which is comparable to the improvement by the direct contact reactor (20 to $27 \%$ ). Based on the quantitative analysis the closed interval design is selected as the better design as it provides a better, stable and linear performance in comparison to the open interval reactor for the operating conditions explored. 


\subsection{Scale up}

The potential scalability of the closed interval design was studied in terms of increasing the diameter of the tubing used. With the same dimensions of the plate (determined by the diameter of the ultrasound transducer), the maximum size of tubing that can be accommodated was determined to be $3.2 \mathrm{~mm}$ OD tubing, which corresponds to an ID of 2 $\mathrm{mm}$. This tubing cannot be arranged as the $0.8 \mathrm{~mm}$ ID tubing as it has a larger bending radius (almost twice as much), therefore instead of going from the first to the second channel the tubing passed from the first to the fourth. The complete arrangement of the tubing is as shown in Figure 11. It is to be noted here that due to this difference in the arrangement, the tubing length increased from $58 \mathrm{~cm}$ to $70.6 \mathrm{~cm}$. The other design characteristics are similar to the $0.8 \mathrm{~mm}$ ID tubing reactor.

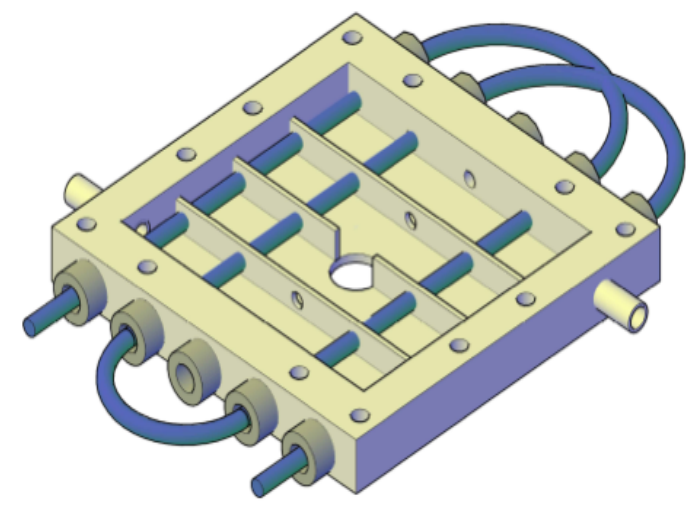

Figure 11: The hybrid closed interval reactor of tubing with ID $2 \mathrm{~mm}$.

Like the previous designs, the scaled up reactor was first examined qualitatively. At silent conditions, when pumping the two-phase system through the $2 \mathrm{~mm}$ ID tubing, even though it starts out as a segmented flow, at a distance of 2 to $3 \mathrm{~cm}$ from the T-junction the dispersed phase detaches itself from the top side of the tubing comparable to as shown in Figure 12 (a). The dispersed phase no longer flows through as a cylindrical bubble with semi-spherical ends but as an ellipsoid. This decoupling from upper limits of tube may be disadvantageous as research by Kashid et al., 2005 has shown that the liquid film formed between slugs (the dispersed phase) and walls of tube contributes to internal mixing. Therefore, the mass transfer may be limited by this elliptical formation. 

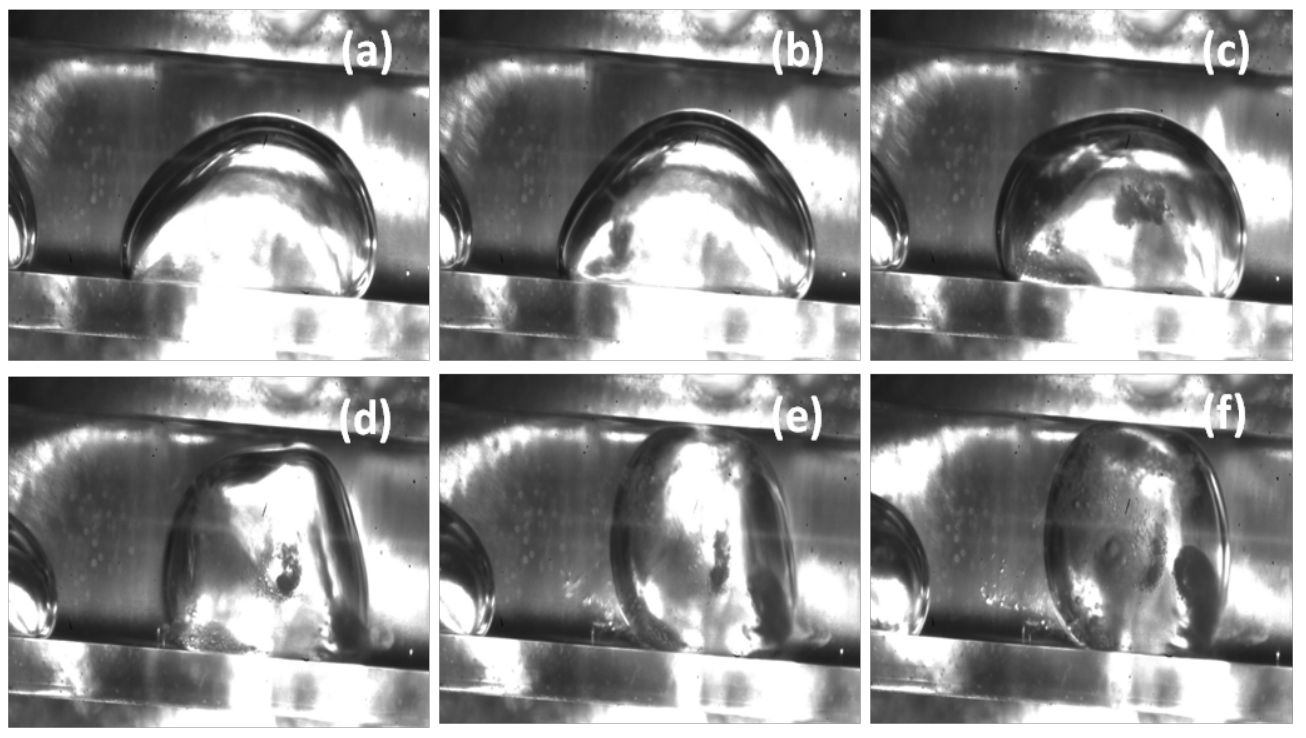

Figure 12: Change of the ellipsoidal bubble back to segmented flow on sonication for $2 \mathrm{~mm}$ ID tubing( a to $\mathrm{f} 1.133 \mathrm{~s}$ ). Images were taken between the first and second interval perpendicular to flow direction

When such a system is sonicated, the following behaviour is observed. First, the initiation mechanism is via cluster formation. It is interesting to note that the cluster acts on the side of the ellipsoid that is in contact with the tubing. The complete emulsification is preceded by a push of the deformed ellipsoid slug back to its original state of a segmented flow as shown in Figure 12. The ultrasound maintains the dispersed phase in a segmented flow regime throughout the sonicated zone. This can be advantageous for scale up of a mass-transfer limited process such as the extraction process, because the segmented flow regime is maintained to impart good mixing due to internal circulation (Dessimoz et al., 2008; Kashid and Agar, 2007; Xu et al., 2013). There are no additional changes observed like dynamic size change in the scaled up version.

To quantitatively examine the effectiveness of the scaled up version, the experiments are conducted with the hydrolysis reaction. The operating parameters are kept similar as the original version even though the scales are different, as the region of sonication is kept constant. The flow rates for the $2 \mathrm{~mm}$ design are selected for similar ranges of residence time and of Reynolds number (i.e., between 8 and 72). The results obtained are shown in comparison to the $0.8 \mathrm{~mm}$ design in Figure $13(\mathrm{a})$. 


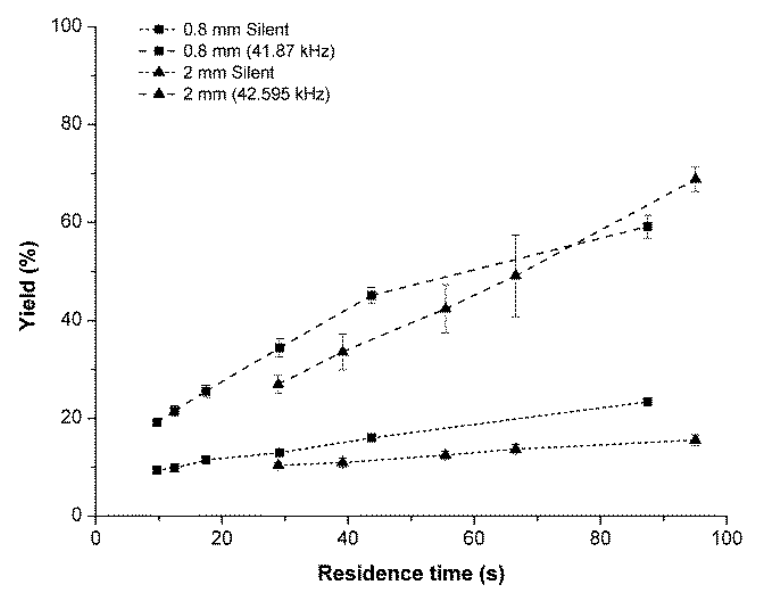

(a)

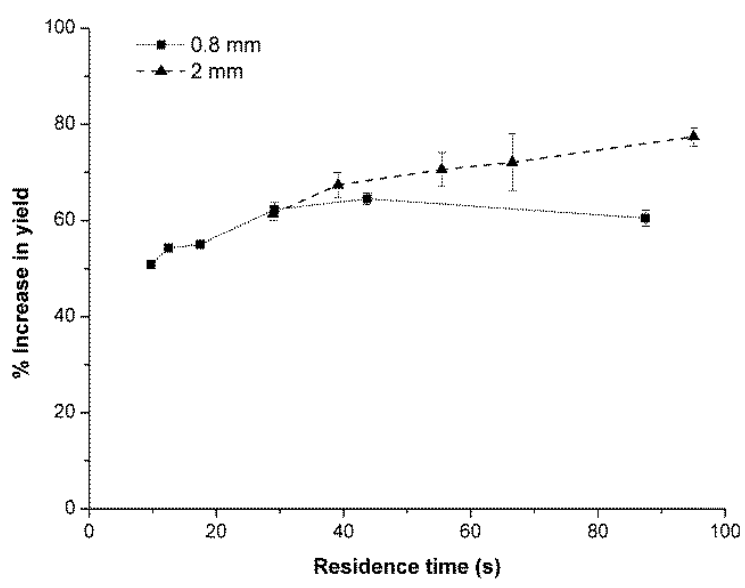

(b)

Figure 13: Variation of yield (\%) with residence time for change of tube diameter at a frequency of $41.4 \mathrm{kHz}$, amplitude of 590 $\mathrm{mV}$ and net applied power of $20 \mathrm{~W}$. (a) Absolute values obtained (b) relative increase in yields to silent condition

The silent conditions are compared first due to the difference in flow pattern. As shown in Figure $5(\mathrm{a})$ the yields obtained are less for the $2 \mathrm{~mm}$ than $0.8 \mathrm{~mm}$, which is understandable as the interfacial area per volume unit decreases with increasing diameters. Comparing the sonicated values, smaller yields are observed compared to the $8 \mathrm{~mm}$ yields for lower residence times of less than $70 \mathrm{~s}$. At higher residence time, the $2 \mathrm{~mm}$ design gives similar or better yields. When examining the yields relatively to their silent conditions (figure 13 (b)), the $2 \mathrm{~mm}$ gives better performance than the $0.8 \mathrm{~mm}$ design. The percentage increase in yield for the $2 \mathrm{~mm}$ design was estimated as being in the same range of between 65 to $75 \%$ as the interval contact design. The interval design gave the highest yields of all designs estimated for similar operating conditions. This yield improvement is interpreted as resulting from lack of dispersed phase coalescence during sonication in the $2 \mathrm{~mm}$ design, which maintains its linearity with respect to residence times. This confirms that lowering yields at higher residence times for the $0.8 \mathrm{~mm}$ design results from coalescence, which may be undesirable for extraction processes. 


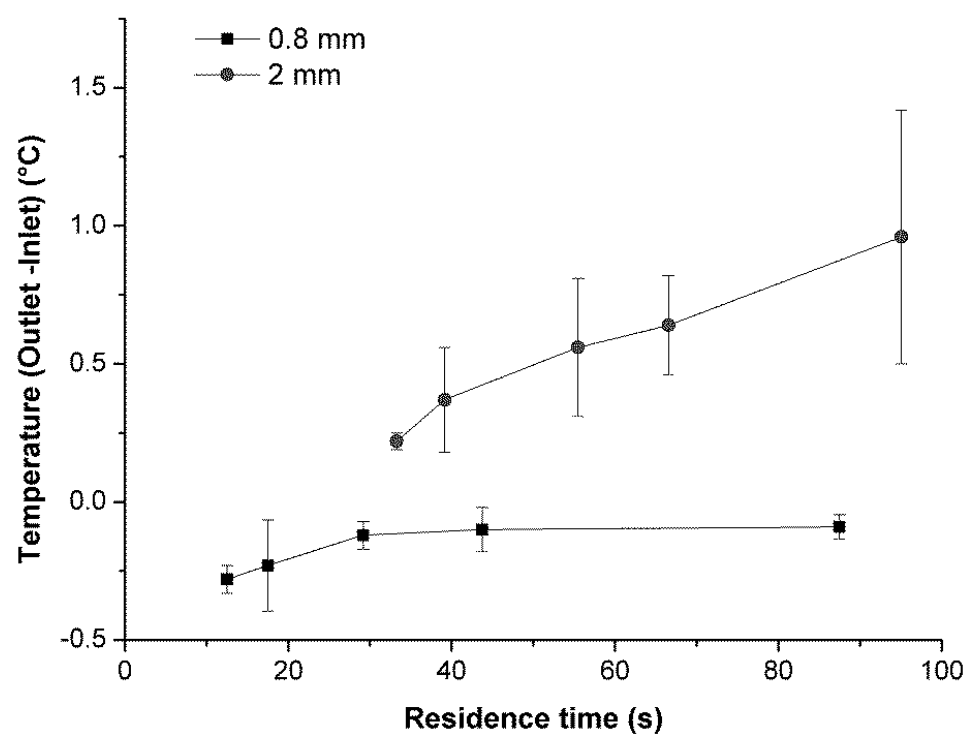

Figure 14: Variation of temperature difference with residence time for the different tubing diameters used in the closed interval reactor at a frequency of $41.4 \mathrm{kHz}$, amplitude of $590 \mathrm{mV}$ and net applied power of $20 \mathrm{~W}$.

To understand why the relative yields are better for a design that in silent conditions would have a lower interfacial area per unit volume, the temperature difference between the inlet and the outlet of the reactor plate were measured using water and compared as shown in Figure 14. The temperature differences obtained are indeed low (less than $2^{\circ} \mathrm{C}$ ) in comparison to the direct-contact designs (in the range of $4-12{ }^{\circ} \mathrm{C}$ ) indicating a degree of temperature control, due to this design's similarity in heat transfer with the indirect contact design. From Figure 14, it is clear that the $2 \mathrm{~mm}$ exhibits larger temperature differences for the same residence times. The result obtained offers two possible conclusions, i.e. that either the $2 \mathrm{~mm}$ design has a better utilization of the ultrasonic energy (by considering the temperature difference to be a reference of the calorimetric power transferred) or that the increase in temperature contributes to increased yield as this is a temperature-sensitive reaction. As seen in a previous publication (John et al., 2016b), the second assumption is unlikely to bring about a $10 \%$ increase in yield as a temperature increase of more than $10^{\circ} \mathrm{C}$ is required to do so. To further support the claim of efficient use of the ultrasound in the $2 \mathrm{~mm}$ reactor, the volumetric mass transfer coefficient $\left(k_{1} a\right)$ is evaluated using the Ramshaw \& Burns method (Burns and Ramshaw, 2001). The method of estimation is detailed out in a previous publication (John et al., 2016a). The results obtained are shown in Figure 15. 


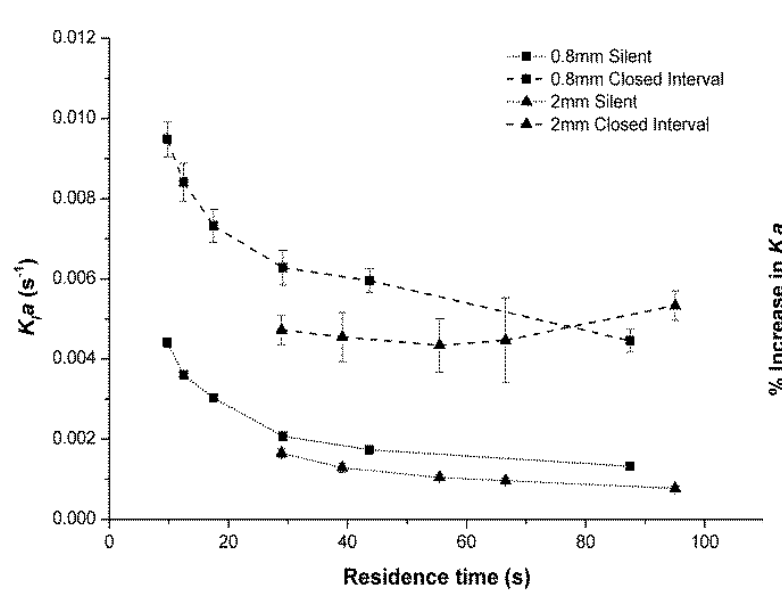

(a)

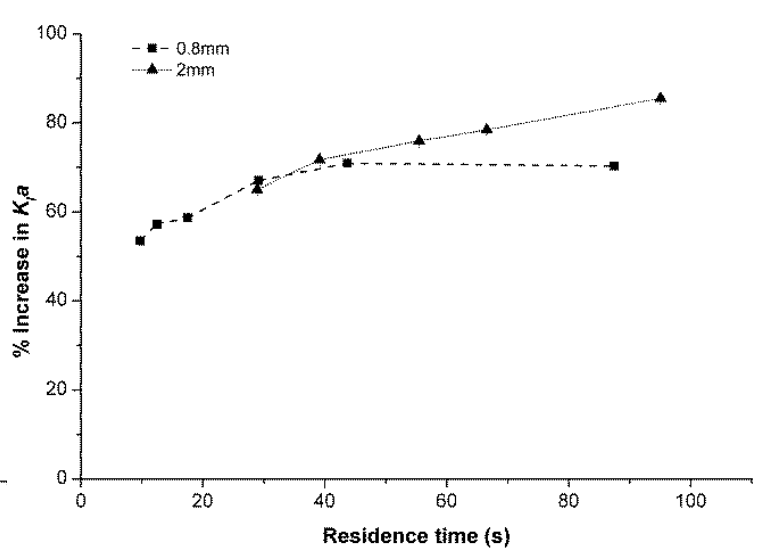

(b)

Figure 15: Variation of volumetric mass transfer coefficient with residence time for change in diameter at a frequency of 41.4 $\mathrm{kHz}$, amplitude of $590 \mathrm{mV}$ and net applied power of $20 \mathrm{~W}$ (a) absolute values, and by (b) relative improvement

The ka values show a similar trend as the yield data and are represented in absolute (Figure 15 (a)) and relative terms (Figure 15 (b)). The absolute values of the $\mathrm{K}_{\mathrm{l}}$ a for the $2 \mathrm{~mm}$ design show a slow increase at the higher residence times but this is not so as it can be attributed to be well within the error range of the experiment. The relative data show similar values in the residence times less than $45 \mathrm{~s}$ but at residence times higher than that value the results diverge, resulting in an advantage of the $2 \mathrm{~mm}$ design over the $0.8 \mathrm{~mm}$ design. This behaviour leads to substantial improvements upon sonication, as in silent conditions the $2 \mathrm{~mm}$ scale exhibits lower values of volumetric mass transfer coefficients in comparison to the $0.8 \mathrm{~mm}$ scale. With sonication volumetric mass transfer coefficient for $2 \mathrm{~mm}$ design increases by 14 to $20 \%$, compared to the $0.8 \mathrm{~mm}$ design. This further supports the possibility that the $2 \mathrm{~mm}$ even with the lower interfacial area per unit volume provides improved mass transfer characteristics, making it a better scale for the hybrid contact reactor.

\section{Conclusion}

A method for combining temperature control with the direct contact method of transferring ultrasound is realised with the help of a new design, which is termed the hybrid contact reactor. Two variations of this design were developed in relation to the positioning of the tubing at the direct contact elements with the same design characteristics as the direct contact intervals with a $0.8 \mathrm{~mm}$ ID tube. This was tested quantitatively with the hydrolysis of $p$-nitrophenyl acetate. At lower flow rates or at high residence times (> $45 \mathrm{~s}$ ) the performances were hindered by coalescence brought about by the presence of the intervals. However, at lower residence times ( $<45 \mathrm{~s}$ ) the improvement in yield was similar for both hybrid designs, and comparable to the direct contact design. Among the two variations of the hybrid design the closed interval 
variation was selected for scale up as it provided a more stable and distinct improvement in comparison to the open interval design. The reactor was scaled up 10 times in volume with a using a $2 \mathrm{~mm}$ ID tube. The scale up design showed better relative performance than the original version at $0.8 \mathrm{~mm}$ scale as there was no coalescence behaviour to inhibit the mass transfer during sonication. This improvement was assessed and verified by estimating the temperature difference for calorimetric reference and the volumetric mass transfer for mass transfer characteristics. The relative improvements in the yields were in the range of the interval contact design, but now at a 10 times larger throughput scale.

\section{Acknowledgement}

The research leading to these results has received funding from the European Community's Seventh Framework Programme (FP7/ 2007-2013) under grant agreement nNMP2-SL-2012309874 (ALTEREGO).

\section{References}

Ahmed, B., Barrow, D., Wirth, T., 2006. Enhancement of Reaction Rates by Segmented Fluid Flow in Capillary Scale Reactors. Adv. Synth. Catal. 348, 1043-1048. doi:10.1002/adsc.200505480

Ahmedomer, B., Barrow, D., Wirth, T., 2008. Effect of segmented fluid flow, sonication and phase transfer catalysis on biphasic reactions in capillary microreactors. Chem. Eng. J. 135, S280-S283. doi:10.1016/j.cej.2007.07.017

Aljbour, S., Tagawa, T., Yamada, H., 2009. Ultrasound-assisted capillary microreactor for aqueousorganic multiphase reactions. J. Ind. Eng. Chem. 15, 829-834. doi:10.1016/j.jiec.2009.09.008

Burns, J.R., Ramshaw, C., 2001. The intensification of rapid reactions in multiphase systems using slug flow in capillaries. Lab Chip 1, 10-15. doi:10.1039/b102818a

Cravotto, G., Cintas, P., 2012. Harnessing mechanochemical effects with ultrasound-induced reactions. Chem. Sci. 3, 295. doi:10.1039/c1sc00740h

Cravotto, G., Cintas, P., 2009. Molecular self-assembly and patterning induced by sound waves. The case of gelation. Chem. Soc. Rev. 38, 2684-2697. doi:10.1039/b901840a

Cravotto, G., Cintas, P., 2006. Power ultrasound in organic synthesis: moving cavitational chemistry from academia to innovative and large-scale applications. Chem. Soc. Rev. 35, 180-96. doi:10.1039/b503848k

Dessimoz, A.L., Cavin, L., Renken, A., Kiwi-Minsker, L., 2008. Liquid-liquid two-phase flow patterns and mass transfer characteristics in rectangular glass microreactors. Chem. Eng. Sci. 63, 4035-4044. doi:10.1016/j.ces.2008.05.005

Di Miceli Raimondi, N., Prat, L., Gourdon, C., Tasselli, J., 2014. Experiments of mass transfer with liquid-liquid slug flow in square microchannels. Chem. Eng. Sci. 105, 169-178. doi:10.1016/j.ces.2013.11.009 
Fernandez Rivas, D., Cintas, P., Gardeniers, H.J.G.E., 2012. Merging microfluidics and sonochemistry: towards greener and more efficient micro-sono-reactors. Chem. Commun. (Camb). 48, 1093547. doi:10.1039/c2cc33920j

Fernandez Rivas, D., Kuhn, S., 2016. Synergy of Microfluidics and Ultrasound. Top. Curr. Chem. 374, 70. doi:10.1007/s41061-016-0070-y

Gogate, P.R., Pandit, A.B., 2004. Sonochemical reactors: scale up aspects. Ultrason. Sonochem. 11, 105-17. doi:10.1016/j.ultsonch.2004.01.005

Hübner, S., Kressirer, S., Kralisch, D., Bludszuweit-Philipp, C., Lukow, K., Jänich, I., Schilling, a, Hieronymus, H., Liebner, C., Jähnisch, K., 2012. Ultrasound and microstructures--a promising combination? ChemSusChem 5, 279-88. doi:10.1002/cssc.201100369

John, J.J., Kuhn, S., Braeken, L., Gerven, T. Van, 2016a. Ultrasound assisted liquid-liquid extraction in microchannels-A direct contact method. Chem. Eng. Process. Process Intensif. 102, 37-46. doi:10.1016/j.cep.2016.01.003

John, J.J., Kuhn, S., Braeken, L., Gerven, T. Van, 2016b. Ultrasound Assisted Liquid-Liquid Extraction with a novel Interval-Contact Reactor. Chem. Eng. Process. Process Intensif. 113, 35-41. doi:10.1016/j.cep.2016.09.008

Kashid, M., Kiwi-Minsker, L., 2011. Quantitative prediction of flow patterns in liquid-liquid flow in microcapillaries. Chem. Eng. Process. Process Intensif. 50, 972-978. doi:10.1016/j.cep.2011.07.003

Kashid, M.N., Agar, D.W., 2007. Hydrodynamics of liquid-liquid slug flow capillary microreactor: Flow regimes, slug size and pressure drop. Chem. Eng. J. 131, 1-13. doi:10.1016/j.cej.2006.11.020

Kashid, M.N., Gerlach, I., Goetz, S., Franzke, J., Acker, J.F., Platte, F., Agar, D.W., Turek, S., 2005. Internal circulation within the liquid slugs of a liquid-liquid slug-flow capillary microreactor. Ind. Eng. Chem. Res. 44, 5003-5010. doi:10.1021/ie0490536

Kashid, M.N., Gupta, a., Renken, a., Kiwi-Minsker, L., 2010. Numbering-up and mass transfer studies of liquid-liquid two-phase microstructured reactors. Chem. Eng. J. 158, 233-240. doi:10.1016/j.cej.2010.01.020

Kashid, M.N., Renken, A., Kiwi-Minsker, L., 2011. Gas-liquid and liquid-liquid mass transfer in microstructured reactors. Chem. Eng. Sci. 66, 3876-3897. doi:10.1016/j.ces.2011.05.015

Katou, H., Miyake, R., Terayama, T., 2005. Non-Contact Micro-Liquid Mixing Method Using Ultrasound. JSME Int. J. Ser. B 48, 350-355. doi:10.1299/jsmeb.48.350

Leonelli, C., Mason, T.J., 2010. Microwave and ultrasonic processing: Now a realistic option for industry. Chem. Eng. Process. Process Intensif. 49, 885-900. doi:10.1016/j.cep.2010.05.006

Linares, P., Lazaro, F., Castro, M.D.L.D.E., Valcarcel, M., 1987. Effects of ultrasonic systems irradiation in flow-injection 200, 51-59.

Mason, T.J., 2000. Ultrasound in synthetic organic chemistry 26, 443-451.

Monnier, H., Wilhelm, A.M., Delmas, H., 2000. Effects of ultrasound on micromixing in a flow cell. Chem. Eng. Sci. 55, 4009-4020.

Monnier, H., Wilhelm, a.-M., Delmas, H., 1999. Influence of ultrasound on mixing on the molecular scale for water and viscous liquids. Ultrason. Sonochem. 6, 67-74. doi:10.1016/S13504177(98)00034-0

Okadap, K., Fuseya, S., Nishimura, Y., 1972. Effect of ultrasound on micromixing. Chem. Eng. Sci. 27, 529-535.

Plouffe, P., Roberge, D.M., Macchi, A., 2014. Liquid-liquid flow regimes and mass transfer in various micro-reactors. Chem. Eng. J. 15-17. doi:10.1016/j.cej.2014.10.072 
Suslick, K.S., Doktycz, S.J., Flint, E.B., 1990. On the origin of sonoluminescence and sonochemistry. Ultrasonics 28, 280-290. doi:10.1016/0041-624X(90)90033-K

Xu, B., Cai, W., Liu, X., Zhang, X., 2013. Mass transfer behavior of liquid-liquid slug flow in circular cross-section microchannel. doi:10.1016/j.cherd.2013.01.014

Chem.

Eng.

Res.

Des.

91

1203-1211. 